\title{
VISUAL SCOTOMATA WITH INTRACRANIAL LESIONS AFFECTING THE OPTIC NERVE
}

\author{
BY \\ ALAN J. MOONEY and ADAMS A. MCCONNELL
}

From the Richmond Hospital, Dublin

Central or paracentral scotoma is an occasional manifestation of certain space-occupying intracranial lesions. The reason for the development of this defect is not yet clear.

Traquair (1946) defines a scotoma as " an area of depressed vision within the field margin surrounded by an area of less depressed or normal vision." Either a depressed area or a channel of varying width may connect the scotoma with the periphery or with the blind spot or with both. Extension of a scotoma to the margin of the field is referred to as "breaking through." When such an extension has occurred careful quantitative perimetry will determine to what extent the central defect predominates in the visual field.

Sudden consciousness of failing vision usually indicates a macular defect, and may bring the patient to an ophthalmologist before pallor of the discs has developed. Bitemporal defects are of gradual onset, and as about 120 degrees of the binocular field remain unaffected the patient's orientation is not seriously disturbed. Hence, except in the case of retrochiasmal lesions when pallor of the disc may never appear, patients whose macular vision is good do not present themselves for examination until they have reached the stage when pallor of one or both discs is evident.

\section{Theories Explaining Scotomatous Defects}

Several theories have been put forward to explain scotomatous defects.

\section{Retrobulbar Neuritis}

Foster Kennedy (1916), when referring to the syndrome described by Paton in 1909 and by himself in 1911 stated that this consisted of " the occurrence of a true -retrobulbar neuritis with the formation of a central scotoma and primary optic atrophy on the side of the lesion, together (if the pressure be sufficiently great) with ipsilateral anosmia and papillodema in the opposite eye."

Larsson and Nord (1947) called attention to the "well-known fact" that the clinical picture of retrobulbar neuritis, consisting of failing vision and central scotoma with or without ophthalmoscopic changes, may be caused by pressure of an expanding intracranial lesion upon the optic nerve in its prechiasmal course. Recently Woods (1948) mentions " intracranial lesions-suprasellar tumours and so forth" as being among the more frequent causes of retrobulbar neuritis.

\section{VULNERABILITY OR HYPERSENSITIVITY OF MACULAR Fibres to Pressure}

Jefferson (1945) stated " that we should abandon any further mention of retrobulbar neuritis, a difficult enough pathological concept without invoking it unnecessarily. Clearly the cause of the scotoma and of the optic atrophy is plain compression and most ophthalmologists are agreed about the special vulnerability of the macular fibres." Kestenbaum (1947) stated, "If the optic nerve is compressed for any reason anywhere in the orbit, in the optic canal or within the cranium, almost always a central scotoma develops. The reason for this phenomenon is still questionable, hypersensitivity of the highly-specialized macular fibres or depleted blood supply (Behr, 1935) have been considered most likely." Peter (1931) wrote, "It is well known that the papillo-macular bundle is more susceptible to toxic or to pressure influence than any other parts of the nerve."

The opinions just quoted imply that pressure on any part of the optic nerve will produce a central scotoma. There are, however, many cases in which the optic nerves appear to be subject to pressure and in which there are no defects of central vision.

In the most common type of pituitary tumour the optic nerves appear to be whiter than normal, to be flattened, and to curve round the tumour tightly embracing it. The expanding tumour must have exerted a considerable amount of pressure to produce such distortion, yet a central scotoma in such a case is a rarity. Before the fields have advanced beyond the stage of bitemporal hemianopsia either the maculæ are spared or they are split by a bitemporal hemianopic scotoma. A meningioma of the tuber- 
culum sellæ produces the same distortion of the optic nerves and the same hemianopic defect. It is difficult to reconcile the obvious pressure on the nerves with the absence of central scotoma if the macular fibres are specially vulnerable. Central scotomata of course do occur, but when they occur the relationship of the tumour to the nerves is not that outlined above.

It has been suggested that upward pressure on an optic nerve against the anterior cerebral artery' may be a factor in the production of scotomatous changes. McConnell and Mooney (1938) reported cases in which there was gross notching of the optic nerves by the anterior cerebral arteries and in which there was neither an inferior altitudinal defect nor a scotoma. Fig. 1 illustrates a definite groove in the optic nerve from which the anterior cerebral artery

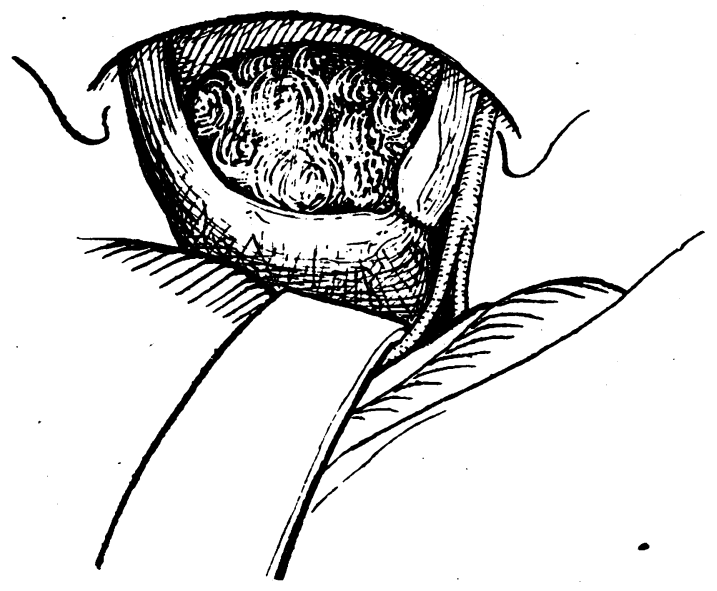

Fig. 1.-Groove on the dorsal surface of the right optic nerve caused by pressure against the anterior cerebral artery.

has been raised, and Fig. 2 shows the visual fields in the same case. Involvement of the macular fibres would appear inevitable in a case of this kind, especially if they were hypersensitive.

Henderson (1939) suggested that acute angulation of the optic nerve against the upper margin of the optic foramen might affect only the macular bundle of fibres and that such an effect could be due to a lateral protrusion of a pituitary adenoma.

Jefferson (1940) depicted acute elevation of the optic nerve close to the optic foramen in a patient with a large extension of a pituitary adenoma into the temporal lobe. In the corresponding eye "vision was lost except for a crescent which ran across the superior temporal and nasal quadrants. Macular vision was lost." The pituitary tumours which are associated with unilateral central scoto- mata appear to be those which insinuate themselves below the nerve close to the optic foramen, thu pushing the nerve upwards.

It is well known that tumours such as olfactory groove meningiomas which exert pressure on the optic nerve immediately behind the optic foramen tend to be associated with central scotomata There is however no constant relationship betweer the incidence of central scotomata and the displace. ment of the nerve. Jefferson (1937) reported two: cases in which an aneurysm was demonstrated just? behind the optic foramen. In each case the nerve was acutely raised and stretched over an aneurysm $\frac{}{2}$. in one case there was a central scotoma, in the othe a hemianopic defect. Perusal of the literature leaves the impression that central scotomata are्s particularly liable to occur when a tumour is sosituated that it either raises or depresses the optic nerve close to the optic foramen. Such pressure at this point pushes the nerve against a rigid? structure, the upper or lower edge of the optie foramen. As at this point the macular fibres forn? an axial core it is necessary to invoke the doctrine of hypersensitivity to explain their involvement.

\section{INTERFERENCE WITH THE BLOOD SUPPLY OF TIE} Optic Nerve

Traquair (1946) wrote, “Anatomical conditions in connexion with the blood supply probably have an important influence as well as special suscegti bility of the macular fibres." He suggested that" pressure on the surface of the nerve might dist the nutrition of the underlying fibres by obstructing their vascular supply, and that conduction intere ference and optic atrophy might be initiated in this way, rather than by the effect of pressure on the् fibres themselves.

Vail (1948) in a review of recent work on the blood supply of the optic nerve states that the intracranial part of the optic nerve is supplied bo branches from the internal carotid, the ophthalmic the anterior cerebral, and the anterior commun cating arteries. "The minute vessels penetrate the pia, pass into the nerve tissue, and form the septa network of the nerve." In the axial portion of the nerve "in which the papillo-macular bundle situated, the septa are thinner and more scarce. (the " end-arteries" of Magitot) than in othes places, and the individual optic-nerve bundles arê thus larger. The nutrition of the papillo-macular bundle is therefore less abundant in the intracraniag part of the optic nerve than elsewhere." The intra canalicular portion of the optic nerve is suppliefs by the ophthalmic artery, and in this portion alsp the pial network is relatively poor (Wolff, 1948).

These anatomical studies suggest that any imf 
2
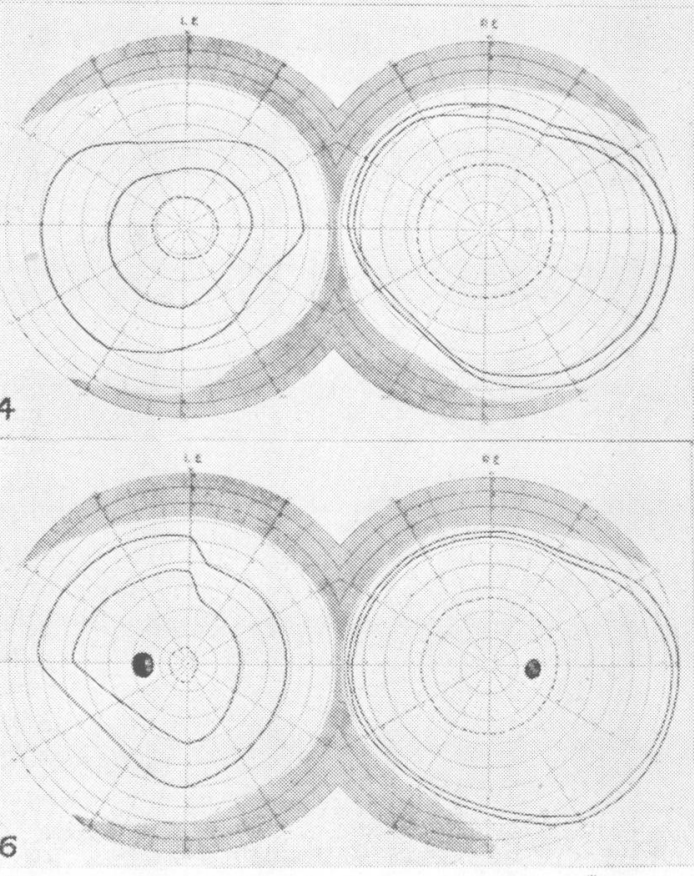
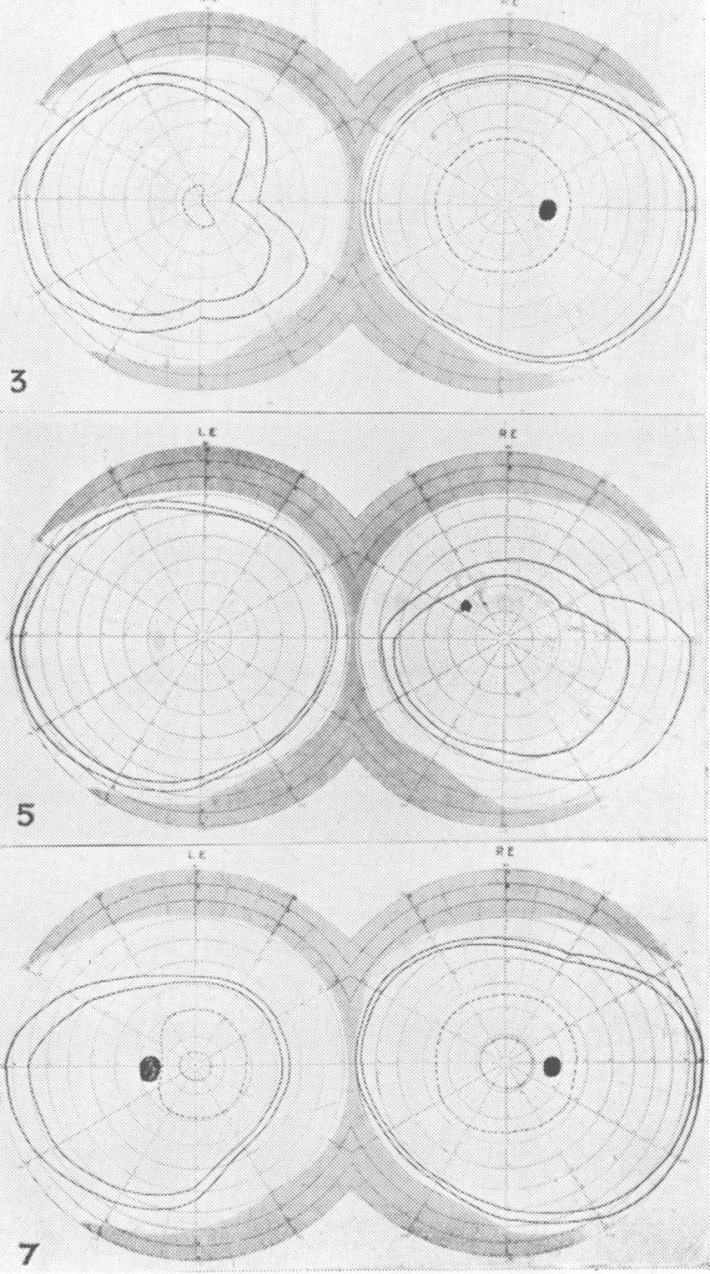

7

FIG. 3.-Visual acuity : right eye, $6 / 5$; left, 6/12. Objects : $3 / 330,1 / 330,2 / 2000$.

Fig. 5.-Visual acuity : right eye, 6/9; left, 6/5. Objects : $3 / 330,1 / 330$.

Fig. 7.-Visual acuity : right eye, $6 / 5$; left, $6 / 6$. Objects : $3 / 330,1 / 330,2 / 2000$ (white) ; 5/2000 (red). 


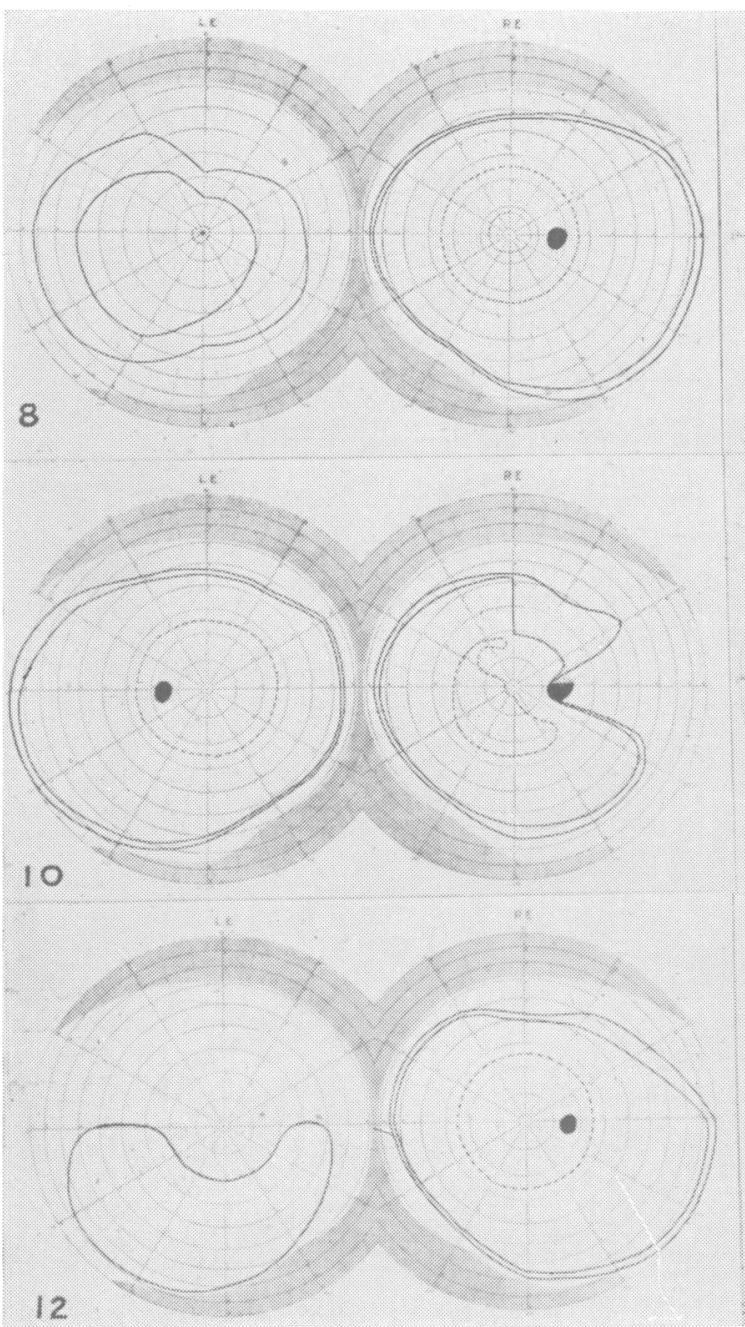

Fig. 8.-Visual acuity : right eye, $6 / 5$; left, $6 / 36$. Objects : $3 / 330,1 / 330,2 / 2000,5 / 2000$ (white), $5 / 2000$ (red).

FIG. 10.-Visual acuity : right eye, $6 / 60$; left, $6 / 6$. Objects : $3 / 330,1 / 330,2 / 2000$.

Fig. 12.-Case 1. Visual acuity : right eye, $6 / 5$; left, perception of light. Objects : 3/330, 1/330, $2 / 2000$; left eye, $20 / 2000$.

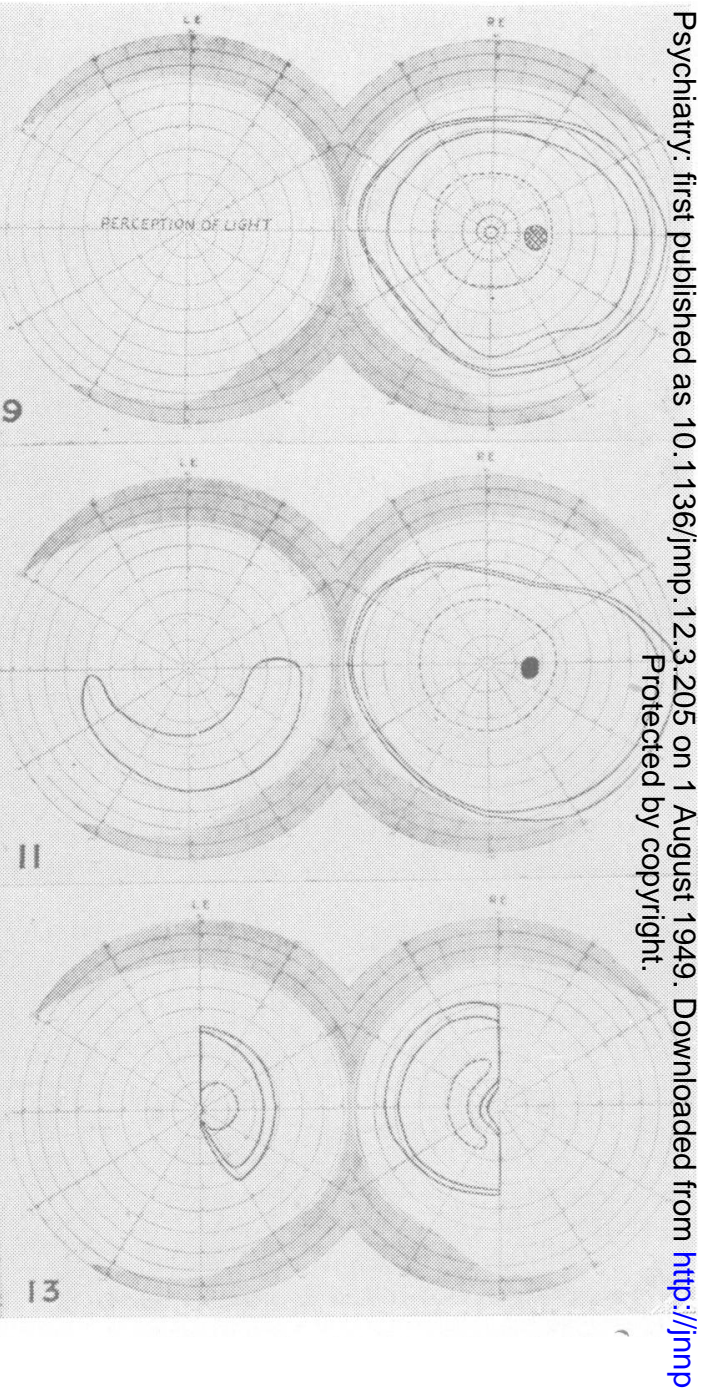

Fig. 9.-Visual acuity : right eye, $6 / 6$; left, perceptio of light. Objects : 20/330,3/330,1/330, 10/2000; 3/2000 (white), 10/2000 (red), 5/2000 (red).

FIG. 11.-Case 1. Visual acuity: right eye, $6 / 3$; left, hand movements. Objects : $3 / 330,1,339$, $2 / 2000$; left eye, $20 / 2000$.

Fig. 13.-Case 2. Visual acuity : right eye, coun fingers at 2 feet ; left, $3 / 60$. Objects : $3 / 330$, $1 / 330,2 / 2000$. 
pairment of the circulation in the intracranial or canalicular portion of the optic nerve would affect the macular bundle more than it would affect other fibres.

It is generally assumed that the field defects following optic-nerve trauma are due to tearing of the pial vessels. As the ischæmia in these cases might not be unlike that due to pressure on the pial vessels from without by a swelling such as a tumour, a short account of the field defects following trauma appears to be relevant.

Hughes (1943) said that he was coming more and more to believe that most field defects were, in fact, due to embarrassment of the vascular supply of the nerve or chiasma as the case might be. "Perhaps it might be possible to explain the diminished conduction in the nerve fibre on a vascular basis, whereas it would be difficult to do so on the basis of direct compression of the fibre." He (1945) states that there are two features in the typical group of neld defects following traumatic lesions of the optic nerve; one, a constant feature, is an inferior altitudinal defect; the other is general depression. In one case only of seventeen reported by this author was a " pericentral" scotoma present.

Turner (1943), in a study of thirtythree cases, found that the visual field defects after partial injury to the optic nerve fall into two main groups, "The first in which a scotoma is the leading feature, the second in which a peripheral sector defect is outstanding." In the scotomatous group there were eight cases, in three of which the scotoma was pericentral, in four paracentral, and in one cæcocentral. In Turner's thirty-three cases there were therefore only three with a central scotoma. Whatever the changes in the peripheral field it seems correct to say that a central scotoma is not a common feature in traumatic lesions of the optic nerve. In Mooney's (1945) series of eight cases of optic nerve injury (Figs. 3 to 10 inclusive) the visual defects were predominantly peripheral, and in the only two cases (Figs. 8 and 10) with macular involvement the retinal arteries were narrower than those of the opposite eye. The latter finding suggests that pericentral defects are due to involvement of the recurrent branch of the central artery of the retina.

As involvement of the pial branches of the ophthalmic artery by trauma does not affect the papillo-macular bundle with any constancy, it is not clear why local involvement of these vessels by pressure from a tumour should have that effect. Traquair pointed out that it was not the position of the tumour but the position of damage to the circulation that would produce field changes.

The possibility that the blood supply of the optic nerve might be impaired by pressure on the ophthalmic artery itself occurred to one of us (A.M.) when inspecting an arteriogram in the following case :

Case 1.-A man aged 28 years was admitted to the Richmond Hospital, Dublin, on Dec. 4, 1946. In July 1945 he had had an attack of severe pain in the left eye. This continued for a few days. In March 1946 he had

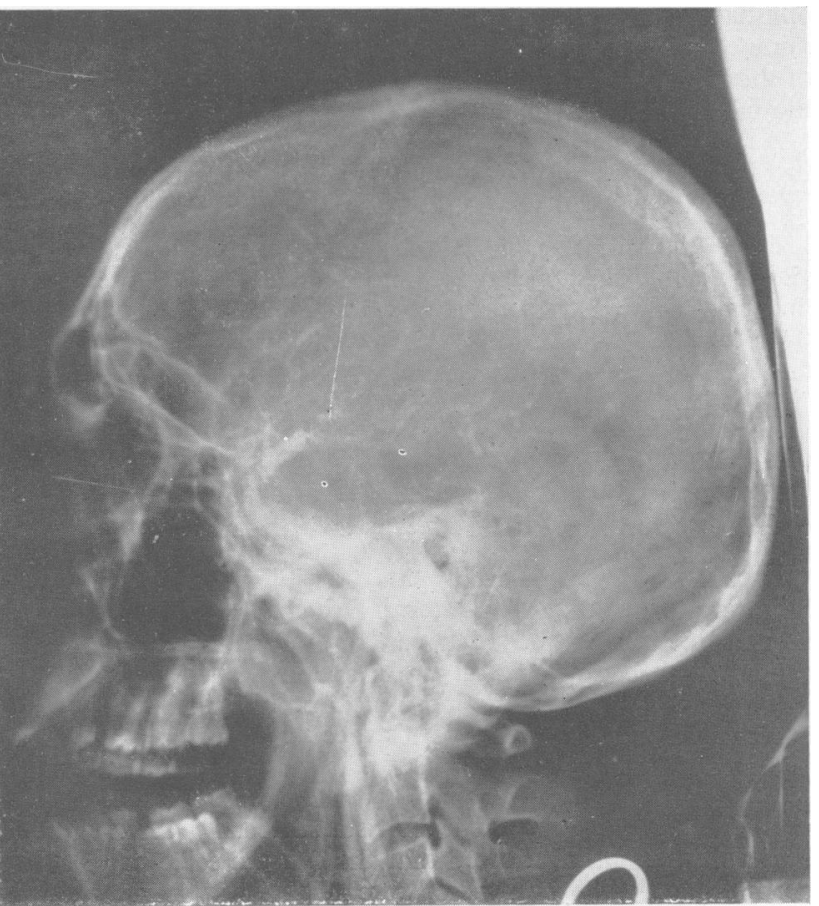

FIG. 14.-Case 1. Arteriogram of the left internal carotid artery showing widening of the carotid curve.

a similar attack and then he noticed that the vision of the left eye was impaired. From that time vision of this eye deteriorated steadily.

The patient was a typical acromegalic. Radiographic examination revealed grcss destruction of the pituitary fossa with disappearance of the clinoid processes and the roof of the sphenoidal sinus. General neurological examination was negative.

Dec. 9, 1946.-The findings on ophthalmic examination were : visual acuity in right eye $6 / 6$ (Snellen) ; left eye, hand movements only; discs normal. The left pupil reacted sluggishly to light. Perimetry showed slight flattening of the isopters in the upper temporal quadrant 
of the right eye and in the left eye a large central scotoma which had broken through above (Fig. 11). The discrepancy between the gross central defect in the left eye and the minimal peripheral defect in the right, so rare with pituitary adenomas, suggested the possibility of an aneurysm, in spite of the characteristic clinical features. Perabrodil was injected into the left common carotid artery. The arteriogram did not reveal an aneurysm but it showed the curve of the carotid syphon to be greatly modified (Fig. 14). The distal curve appeared to be widely opened out and the supraclinoid portion of the artery to be higher than normal. The appearance suggested that the tumour had extended laterally inside the natural curve of the artery. It seemed that such a displacement of the internal carotid artery might possibly kink or partially obstruct the ophthalmic artery at its origin or occlude some of the minute branches which it gives off in this region. Some of these branches may be destined to supply a section of the papillo-macular bundle.

As this patient was submitted to deep radiation therapy there was no opportunity to verify the surmise by direct inspection.

Oct. 11, 1948.-Ophthalmic examination showed visual acuity, right eye $6 / 5$ (Snellen); left eye counts fingers close to his face. Right field unchanged; left field, large central scotoma with normal lower peripheral field to hand movements (Fig. 12).

-It so happened that when this patient was under observation an operation was performed on another patient with a large central scotoma, and an elevation of the internal carotid artery was demonstrated.

Case 2.-A man aged 33 years was admitted to the Richmond Hospital, Dublin, on Dec. 4, 1946. Nine months previously he had begun to suffer from headaches, which became steadily more frequent and severe. About the same time he noticed that his sight was becoming poor. The skin of his face was smooth and hairless. No genital deformity was noted. General examination was negative and there were no abnormal neurological signs apart from the visual defects. Radiographic examination showed that the dorsum sellæ had been eroded and that there were slight but definite supra- and intra-sellar calcification.

Ophthalmic Examination.-The visual acuity was : right, counting fingers at 2 feet; left, 3/60 (Snellen). The right disc was normal, the left pale with clear margins and normal vessels. Perimetric examination revealed bitemporal hemianopsia for $3 / 330,1 / 330$, and $5 / 2000$, white, with a large central scotoma on the right side and a small one on the left for these visual angles (Fig. 13).

Dec. 6, 1946.-An operation for right frontal craniotomy was performed. When the right optic nerve was exposed it was seen to rise abruptly from the optic foramen and to pass to the temporal side. Further exposure showed that its acute elevation was due to a soft cystic tumour which also filled the pituitary fossa and appeared to overflow the left dorsal surface of the chiasma (Fig. 15). Aspiration of $6 \mathrm{ml}$. of oily fluid allowed the right optic nerve to become relaxed and flat, and the right carotid artery which had been cof cealed by the displaced nerve was seen to lie on the upper aspect of the cyst. The left optic nerve w displaced laterally but was not grossly elevated. wide opening was made in the cyst wall and a consider able amount of gelatinous tumour was removed with curette. The tumour extended backwards under the chiasma but no attempt was made to discover its posteriēr. limit. The Pathological report diagnosed a cranispharyngioma.

Jan. 15, 1947.-Ophthalmic examination showed marked recovery in the fields. Both presented the appearance of moderate general depression for t $\bar{R}$ larger isopters with bitemporal hemianopsia for 10/200 red (Fig. 19).

Comment.-The gross central scotoma in thips case seemed to be unrelated to the bitemporal

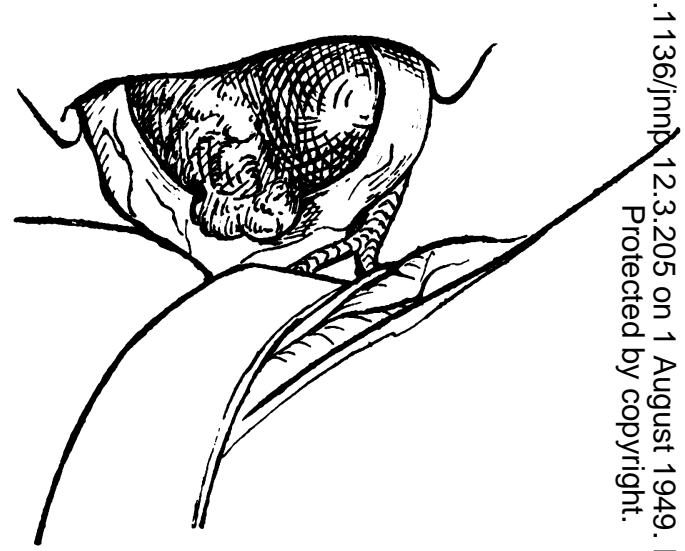

FIG. 15.-Case 2. Craniopharyngioma with large cyt elevating the right optic nerve.

hemianopsia. The latter could be explained pressure on the medial aspects of the optic nerves or by involvement of the chiasma, but the formos demanded some other explanation. The association in two cases of a large central scotoma with same kind of variation in the appearance of the internal carotid artery led to a study of the anatoriay of the opthalmic artery.

The ophthalmic artery comes off the medial side of the upper aspect of the internal carotid arteisy at its last bend. It then passes across the convexify of the artery between it and the optic nerve before coming to lie on the lateral side of the nerve (Fig. 19).

The relationships of the internal carotid arte the ophthalmic artery, and the optic nerve close the optic foramen appear to be such that any seved pressure on the optic nerve from above would necessarily be transmitted to the ophthalmic arter. and any pressure on the internal carotid artefry 
from below would press the ophthalmic artery against the nerve and both against the falciform fold which forms the upper edge of the optic

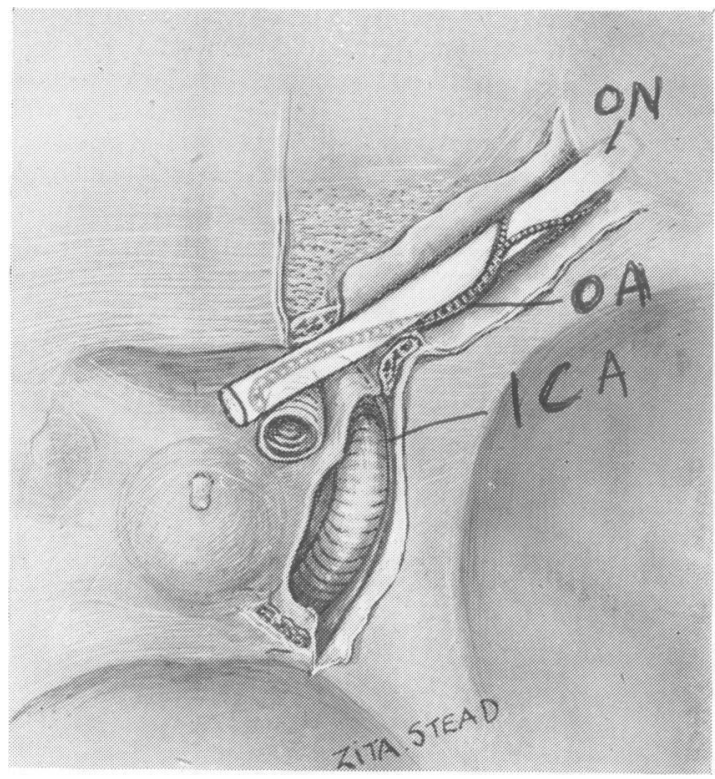

FIG. 16.-From a dissection to show the origin and course of the ophthalmic artery. The nerve is represented as transparent. The roof of the optic canal has been removed and the dural sheath opened. right and reacted sluggishly to light. Visual acuity was : right, 6/5 (Snellen); left, counting fingers at one metre. The right disc was normal ; the left was pale with clear margins and normal vessels.

Oct. 24, 1937.-The right eye was normal. The left eye had a large cæco-central scotoma for $40 / 2000$, breaking through for $5 / 2000$ and $2 / 2000$, white. There was slight general depression (Fig. 20).

Oct. 28, 1937.-At operation a large left transfrontal exposure was made. The optic nerve was found running in a normal direction but both it and the internal carotid artery were elevated upwards about $\frac{1}{2} \mathrm{~cm}$. behind the optic foramen and seemed to be riding over the surface of a tumour. The dura of the middle fossa was found to be lifted up to the level of the sphenoidal ridge (Fig. 17).

Incomplete removal of meningiomatous tissue was effected from the left middle fossa. Some tumour was scooped out from beneath the left optic nerve but at the conclusion of the operation the nerve and vessel had not assumed normal levels.

Nov. 14, 1937.-Examination showed slight left-sided exophthalmos. The fundi were unchanged. Visual acuity was : right, 6/5 (Snellen); left, 3/60 (Snellen).

The visual field of the right eye was normal. In the left eye the cæco-central scotoma for $40 / 2000$, white, had disappeared. There was some recovery in the $5 / 2000$, and $2 / 2000$ isopters. The general depression was less marked (Fig. 21).

In the two verified cases just reported (Cases 2 and 3) the maximum pressure on the optic nerve and internal carotid artery seemed to be exerted

foramen. In either event what would be seen during the operative exposure would be the nerve depressed by something above it or the nerve raised and stretched by something below it. In both cases the ophthalmic artery would be concealed by the optic nerve. We had noted an elevation of the internal carotid artery on previous occasions but had not attached any significance to it as the obvious deformity of the optic nerve seemed to be a sufficient explanation of the scotomatous defect.

The following case is an example :

Case 3. - A man aged 56 years was admitted to hospital on Oct. 22, 1937. He stated that his left ear had become deaf in 1928 and that in 1935 the left eyelid drooped and the eye turned upwards. Four months before admission he found that the vision of the left eye had nearly disappeared. There had been a complete absence of pain or numbness.

On examination there was ptosis of the left eyelid with mild exophthalmos; the left pupil was smaller than the

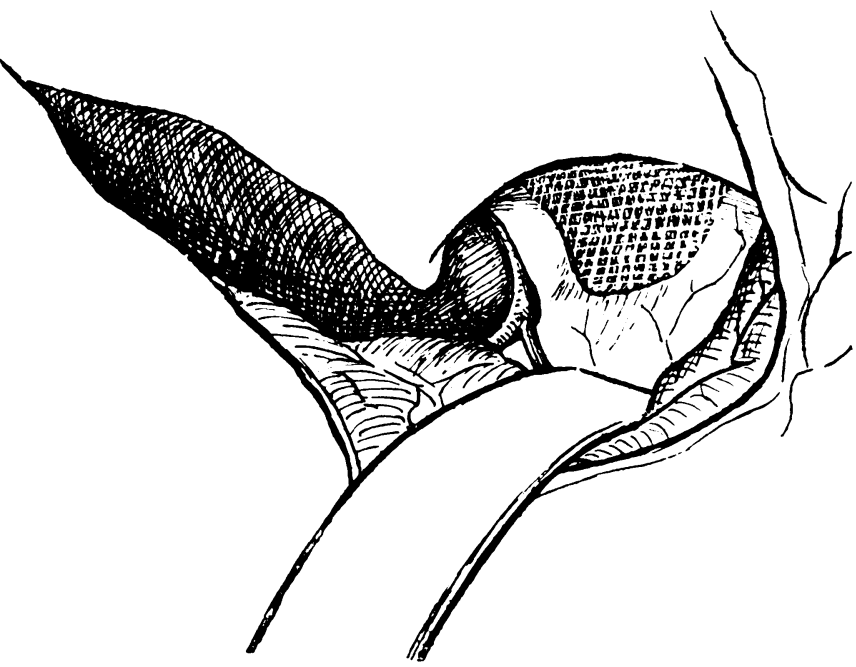

FIG. 17.-Case 3. Meningioma of the middle cranial fossa passing medially under the internal carotid artery and the left optic nerve.

close to the optic foramen, the tumour undermining the anterior clinoid process. In the unverified case 
the widening of the arterial curve suggests that the tumour had grown into the anterior depth of its concavity. It may be that such a forward extension of the tumour is necessary if a central scotoma is to be produced, whether or not obstruction of the ophthalmic artery be an intermediary factor. In two patients with moderate temporal extensions of pituitary adenomata the tumour passed under the posterior portion of the optic nerve and the maximum elevation of the internal carotid artery was at its bifurcation. In neither case was there any scotomatous change.

A widening of the carotid curve was demonstrated both by arteriography and operation in another patient with a left lateral protrusion of a pituitary adenoma, but as the left eye was blind it was impossible to state whether or not there had been an early central scotoma.

In the following case a central scotoma was associated with a swelling which raised and stretched the optic nerve just behind the optic foramen. The position of the internal carotid artery had not been recorded.

Case 4.-A man aged 32 years was admitted to the Richmond Hospital on April 27, 1937. Four years previously he had noticed that the vision of the left eye was poor and that he could not see objects if he looked directly at them. Headaches had also begun four years previously. At first they were not severe but they became worse and on admission to hospital they were constant and incapacitating. He had had occasional attacks of "weakness" for the past year. General neurological examination was negative. A radiograph of the skull showed destruction of the left anterior clinoid process with erosion of the sphenoidal ridge beside it. The left optic foramen appeared a little larger than the right.

May 2, 1937.-Ophthalmic examination showed visual acuity to be : right, $6 / 5$ (Snellen); left, counted fingers at 1 metre. The right eye showed odema of the nasal margin of the disc. The left had a pale disc with clear margins and normal vessels.

The visual field of the right eye showed an enlarged blind spot but was otherwise normal. In the left eye there was a large cæco-central scotoma for $3 / 330,1 / 330$ white, with some general depression (Fig. 22). The diagnosis was made of a lesion, probably meningioma or aneurysm in the region of the left optic foramen.

Ventriculography was performed on May 20. A few drops of fluid escaped from the right ventricle under pressure, but no more could be obtained : $20 \mathrm{c} . \mathrm{cm}$. were removed from the left ventricle and replaced by air. The films showed the anterior part of the left ventricle to be displaced from the mid-line. There was no air in the right ventricle or in the third ventricle. At operation a right transfrontal exposure was made. A very large subarachnoid cyst had practically replaced that part of the right frontal lobe which lay in front of the pre-central sulcus. On the floor of the cyst appeared the chiasma and both optic nerves. The left optic nerve was stretched tightly over another swelling about th size of a pea (Fig. 18). There was a suggestion of pulsation in the swelling which was therefore taken te be an aneurysm. A blunt hook was passed along the nerve under the falciform fold and the ligamento divided as far forward as the bone. The latter seemese to have been eroded. This manœuvre released the anterior part of the nerve so that its upward convexity. was not so acute. The operation relieved the headache but did not improve vision.

Nov. 14, 1947.- The field of the right eye was un $\stackrel{\vec{F}}{4}$ changed. The left eye had scotoma for $1 / 330$, breakin through, but otherwise no change (Fig. 23).

In the cases just recorded pressure on the optie्क nerve or on the internal carotid artery was exerted primarily by the growth of a tumour below these structures. In the following case the pressure was exerted from above.

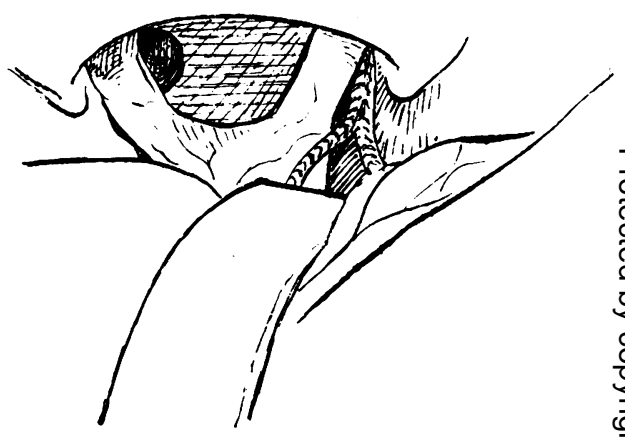

FIG. 18.-Case 4. Presumed aneurysm under the leff optic nerve.

Case 5.-A woman aged 37 years was admitted to the Richmond Hospital on Jan. 15, 1948. Eight month before admission she began to suffer from severe mornin headaches. The severity of these headaches had some@ what diminished before her arrival in hospital, but as they declined the patient became euphoric, expansive and jocular with little appreciation of her illness. Visions began to fail three months before admission. O examination there was complete anosmia but apart from the visual defects no other neurological sign? Ophthalmic examination showed visual acuity to be right, 6/24 (Snellen) ; left, hand movements only. The right disc showed papillœdema of less than 1 diopter the left disc was pale with clear margins and norma vessels.

The visual field of the right eye showed a temporab hemianopic defect for 3/330 and 1/330 white, with smalP macular loss for $2 / 2000$ white, and of the left eye a large central scotoma for hand movements (Fig. 24).

Jan. 26, 1948.-Ventriculography showed displace ment of the anterior portions of both ventricles to the left side. At operation a large meningioma was founq covering most of the right orbital roof and cribriforn $\psi$ 

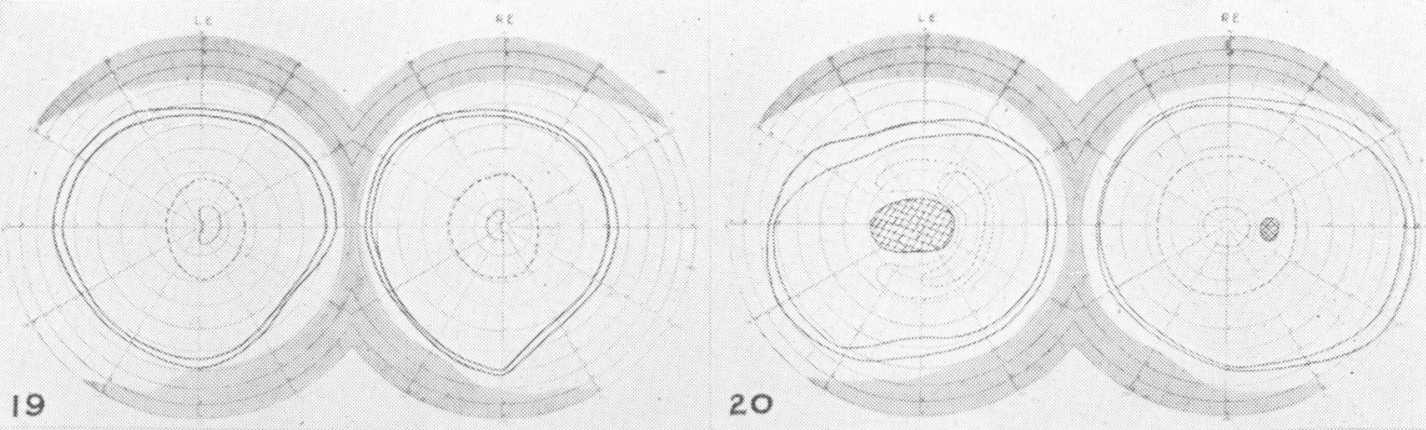

21
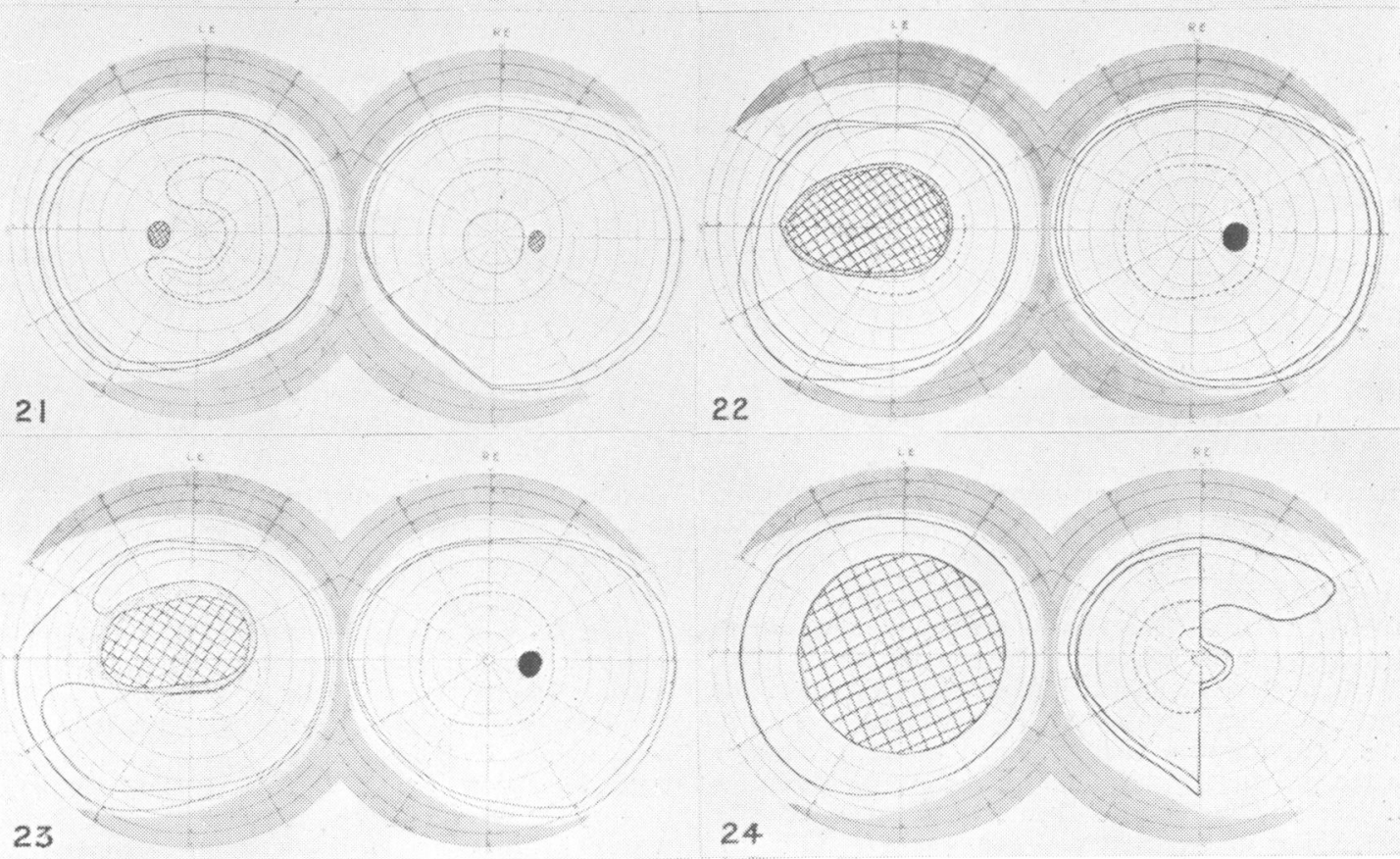

FiG. 19.-Case 2. Visual acuity: both eyes, 6/36. Objects : $3 / 330,1 / 330,2 / 2000$ (white), 10/2000 (red).

Fig. 21.-Case 3. Visual acuity: right eye, 6/5; left, $3 / 60$. Objects : $3 / 330,1 / 330,5 / 2000$, $2 / 2000$ (white), right eye, $5 / 2000$ (red).

Fig. 23.-Case 4. Visual acuity: right eye, 6/6; left, counts fingers at 1 metre. Objects : $3 / 330$, $1 / 330,2 / 2000$ (white), right eye, 10/2000 (red).

FIG. 20.-Case 3. Visual acuity: right eye, 6/5; left, counts fingers at 1 metre. Objects : $3 / 330$, $1 / 330,2 / 2000,5 / 2000$ (white), right eye, 5/2000 (red).

FIG. 22.-Case 4. Visual acuity: right eye, 6/6; left, counts fingers at 1 metre. Objects; $3 / 330$, $1 / 330,2 / 2000$.

FIG. 24.-Case 5. Visual acuity : right eye, 6/24 ; left, hand movements. Objects : $3 / 330,1 / 330$, $2 / 2000$. 


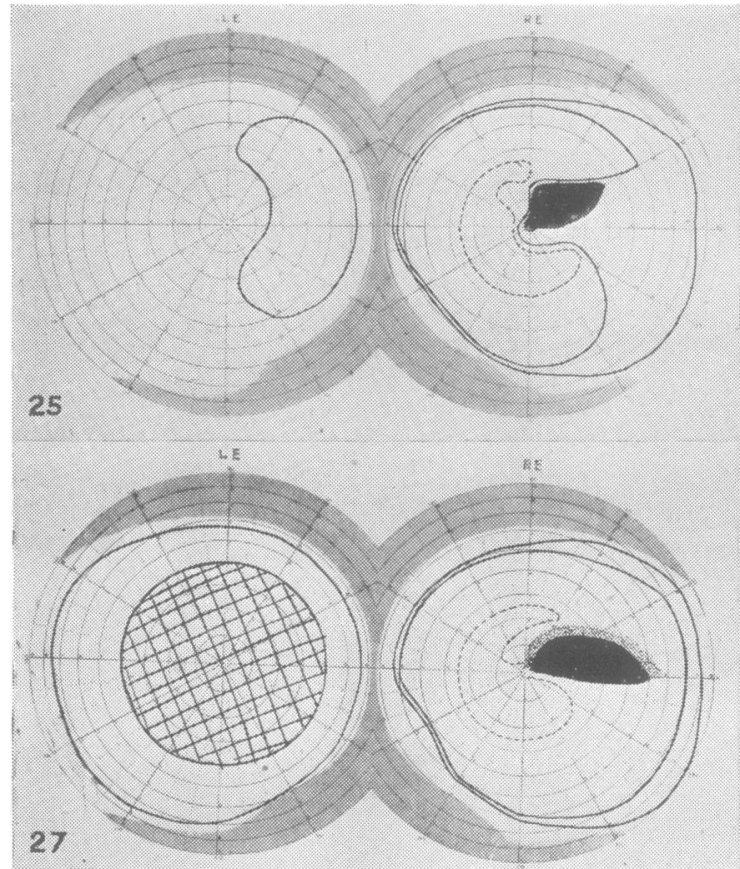

FIG. 25.-Case 5. Visual acuity : right eye, 6/24; left, counts fingers at 1 metre. Objects : $3 / 330$, $1 / 330,2 / 2000$.

FIG. 27.-Case 5. Visual acuity : right eye, 6/18; left, hand movements. Objects : $3 / 330,1 / 330$, 2/2000.

plate. It extended backwards over both optic nervès and across to the left side of the anterior cranial fossa. The tumour was completely removed on the right side but some of its extension to the other side was left in situ. Postoperative charts showed a steady recovery in the peripheral field of the right eye with shrinkage of the residual scotoma. There was no material change in the field of the left eye. (Feb. 15, 1948 : Fig. 25. Mar. 14, 1948 : Fig. 26. May 2, 1948 : Fig. 27. July 7, 1948 : Fig. 28.).

Comment.-It is impossible to say to what extent the tumour had depressed the right optic nerve or had involved the ophthalmic artery. The case is included because of the association of a central scotoma with a tumour affecting the optic nerve close to the optic foramen.

In four of the five cases just recorded the lesions and their relations to the optic nerve were verified at operation ; in Case 1 there was sufficient evidence to support the diagnosis of a pituitary adenoma with a temporal extension. In the following case the optic nerve was not seen but the evidence suggests that pressure had been exerted on its upper surface.

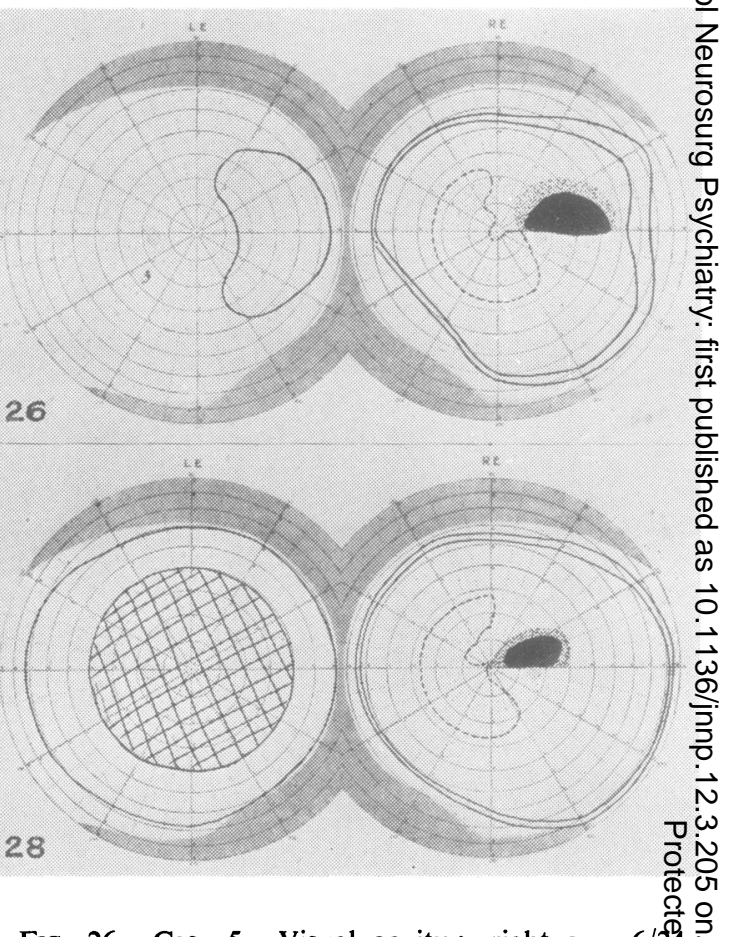

FIG. 26.-Case 5. Visual acuity : right eye, 6/ 29 left, counts fingers at 1 metre. Objects : $3 / 30$ $1 / 3302 / 2000$.

Fig. 28.-Case 5. Visual acuity: right eye, 6/18 left, hand movements. Objects : $3 / 330,1 / 3=0$ 2/2000.

Case 6.-A man aged 38 years in 1925 had sustaine $\$$ concussion and a scalp wound in the right parieta region. He recovered rapidly and resumed work i ten days. In 1936 he began to have attacks of " un consciousness" lasting about fifteen minutes an $\bar{\phi}$ occurring about twice a year. A detailed description of these attacks could not be obtained. In the las week of February 1939 he began to notice some transien blurring of vision. On examination on March 3 th visual acuity was $6 / 6$ (Snellen) in each eye. The right disc was normal ; the upper margin of the left disc was. very slightly blurred. There was no gross defect i $\overline{\mathbf{p}}$ either visual field. One week later there was slight œdema of the upper margin of the right disc, while the left disc was unchanged.

The patient was admitted to the Richmond Hospita on July 19, 1939. General neurological examination was negative, and the skull appeared normal radiog graphically. At ophthalmic examination on Aug. 25 1939, visual acuity of each eye was $6 / 6$ (Snellen). The right disc had papillœdema of 2 diopters; the left had blurring of upper margin. There was paresis of tho left inferior rectus muscle. With the exception of enlarged blind spots, especially the right, the visuag fields were normal. On Aug. 27 visual acuity of the 

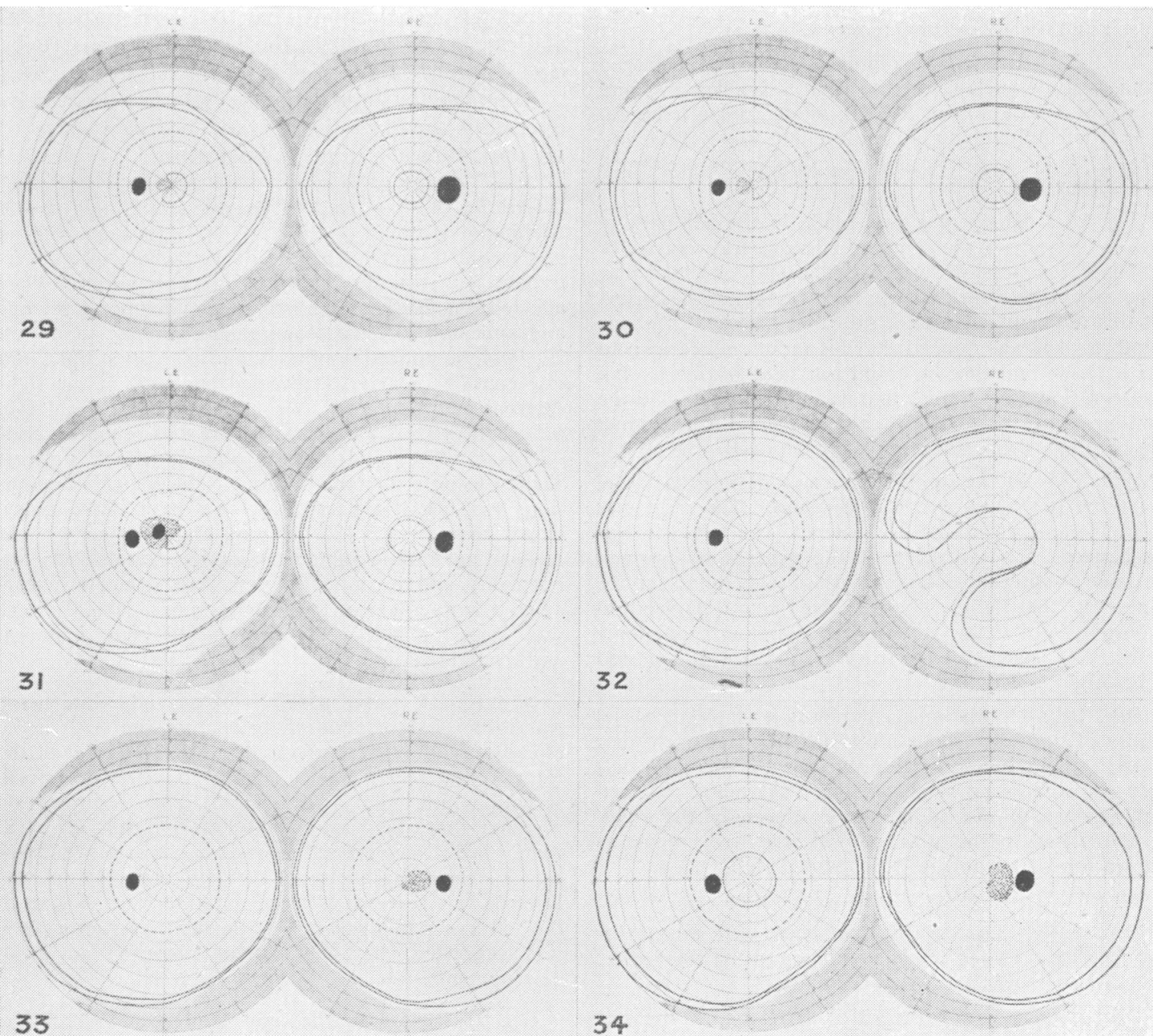

34

FIG. 29.-Case 6. Visual acuity : right eye, 6/6 ; left, $6 / 12$. Objects : $3 / 330,1 / 330,2 / 2000$ (white), $5 / 2000$ (red).

FIG. 31.-Case 6. Visual acuity: both eyes, 6/6. Objects : 3/330, 1/330, 2/2000 (white), 5/2000 (red). Scotoma : 2/2000,5/2000, 10/2000 (white).

FIG. 33.-Case 7. Visual acuity : right eye, 6/9; left, $6 / 5$. Objects : $3 / 330,1 / 330,2 / 2000$.

Fig. 30.-Case 6. Visual acuity : both eyes, 6/6. Objects : $3 / 330,1 / 330,2 / 2000$ (white), 5/2000 (red).

FIG. 32.-Case 7. Visual acuity : right eye, $3 / 60$, left, $6 / 6$. Objects : $3 / 330,1 / 330,2 / 2000$.

FIG. 34.-Case 7. Visual acuity : right eye, 6/6 ; left, $6 / 5$. Objects : $3 / 330,1 / 330,2 / 2000$ (white), 5/2000 (red). Scotoma : 2/2000, 5/2000 (white). 
right eye was $6 / 6$ and of the left $6 / 12$ (Snellen). Papillœedema of the right disc had increased to $3 \frac{1}{2}$ diopters, while the slight blurring of the upper margin of the left disc remained unchanged. Both the left inferior rectus muscle and the left external rectus were weak.

Aug. 27.-Perimetric examination showed enlarged blind spots, especially on the right. The left eye had paracentral scotoma for $2 / 2000$, white, and a temporal sector defect involving fixation point for $5 / 2000$, red (Fig. 29). General neurological examination continued to be negative.

Aug. 29.-An opening was made in the left parietal region just above the lambdoid suture for ventriculography. The bone was very vascular and the ventricular needle met with a hard tumour mass just below the cortex. At this moment the patient said that his right arm was numb, and it was noticed that his mouth was drawn to the left side and that he could not raise his left eyelid. At operation a large bone flap was turned down and the tumour, which proved to be a parasaggital meningioma ( $5 \frac{1}{2}$ by 4 by $4 \mathrm{~cm}$.), was completely removed.

Sept. 17.-At the ophthalmic examination the visual acuity of both eyes was $6 / 6$ (Snellen). The right disc showed papillœdemà of 3 diopters. The left disc was unchanged. The muscles of the eyes were normal. Perimetry showed enlargement of the blind spots, especially the right. The left eye had a paracentral scotoma for $2 / 2000$, white, and temporal hemianopic defect with sparing of the fixation point for $5 / 2000$, red (Fig. 30).

Dec. 10.-Ophthalmic examination showed visual acuity of both eyes to be $6 / 6$ (Snellen). The right disc showed slight blurring of the upper margin. The left disc showed very slight pallor. Perimetry showed enlargement of the blind spots, especially the right. In the left eye paracentral scotoma was more intense as shown for 5/2000 and 10/2000, white (Fig. 31).

March 3, 1940.-Visual fields were normal.

Dec. 1948.- The patient has been well except for an occasional attack of slight tingling in the right arm : consciousness was not lost in these attacks.

Comment.-The paracentral scotoma in the left eye did not show hemianopic characteristics. The presence of this scotoma, together with a disc which was within normal limits in the left eye and papillœdema in the right, led us to expect a leftsided baso-frontal tumour. If ventriculography had not been attempted this would have led to a left-frontal exposure. It would appear in retrospect that the swelling or downward displacement of the left hemisphere in this patient had exerted pressure on the left optic nerve and possibly on the ophthalmic artery under the nerve. Involvement of the third cranial nerve was probably due to a cerebral herniation through the anterior part of the tentorial opening, or into the pituitary fossa. We have noted on other occasions herniation of the posterior end of the gyrus rectus over the sharp dural fold which continues the edge of the tentorium to the anterior clinoid process. Vincent and others (1938) reported two cases of primary optic atrophy associated with meningiomas, one parieto-occipital and one parasaggital in the parietal region. Beck (1947) published a case of the same nature.

Although the following case was not verified by operation the diagnosis of an aneurysm close to the optic foramen seems to be justified.

Case 7.-A man aged 49 years was admitted to the Richmond Hospital on July 5, 1947. In June 1944 he had begun to suffer from headaches which were intermittent at first but gradually became continuous. They $\frac{\bar{c}}{\bar{N}}$ affected chiefly the right frontal and temporal regions and appeared to have a neuralgic character. On Oct. 27,1944 , he noticed double vision for the first time, and $ळ$ examination on Nov. 1 revealed paralysis of the right $\vec{\circ}$ external rectus muscle. Visual acuity was : right eye, $6 / 5$; left eye, $6 / 5$ (Snellen). There was retinal arterio- $\vec{\omega}$ sclerosis. Discs and corneal sensation were normal. S There was no intracranial bruit. Neurological examination was negative except for the sixth nerve palsy.? Blood pressure was $180 / 110 \mathrm{~mm}$. Hg. Neither general $\vec{N}$ medical nor radiographic examination revealed any $a b_{j} \omega$ normality, and the optic foramina were normal in appeas- i ance. Compression of the right common carotid arte was found to relieve the pain in the right temporand 윽 and frontal regions.

Nov. 15.-A partial paralysis of the right third nerve? was discovered in addition to that of the sixth neree already noted. There was subjective anæsthesia of right frontal region and a definite lowering of corne $1 \vec{\circ}$ sensation on that side. Ophthalmic examination revealed that the visual acuity of the right eye had diminished to counting fingers at 1 metre. A large central scotoma breaking through downwards and inwards was revealed by confrontation test. Vision of $\frac{\bar{O}}{\mathrm{O}}$ the left eye remained at 6/5 (Snellen). Arteriography was suggested, but the patient preferred to await developments.

Feb. 21, 1945.-At the ophthalmic examination the 읔 right eye had partial third nerve palsy ; the pupil was slightly dilated and reacted sluggishly to direct illumination; the disc was somewhat pale with clear margins visual acuity $3 / 60$, and central scotoma breaking through downwards and inwards. The left eye was normalo (Fig. 32).

April 14, 1946.-At ophthalmic examination the third nerve palsy had completely disappeared and corneal sensation was normal. Visual acuity of right eye was $6 / 9$ (Snellen), and the visual field showed a scotoma involving the cæco-central region but sparing the $>$ macula (Fig. 33).

The patient was able to continue his work until July 1947, when he returned because the headaches hado increased in severity. On this occasion the right discr was pale with clear margins, and connective tissue on theN surface and the left disc showed papillodema of two 
diopters. The latter finding was attributed to hypertension associated with the arteriosclerosis.

July 9, 1947.- The right common carotid artery was injected with perabrodil. The arteriogram did not demonstrate a characteristic aneurysmal sac, but it showed widening and irregularity of the carotid shadow at the last bend of the vessel. The picture suggested an aneurysm lying both inside and outside the cavernous sinus. The common carotid artery was partly occluded in the neck. This procedure relieved the headache to some extent. Six days later the artery was completely occluded. Headache was severe for two days and then gradually disappeared.

June 20, 1948. - The patient appeared well in every way. Visual acuity was : right, $6 / 6$ (Snellen) ; left, 6/6 (Snellen). In the right eye there was an enlarged blind spot and a temporal paracentral scotoma partly encircling but not involving the fixation point (Fig. 34).

The right disc was pale with clear margins and connective tissue on the surface. The left showed slight œdema of the margins. In both retinæ the arteriosclerotic appearances had become more marked. The blood pressure at this time was $205 / 140 \mathrm{~mm}$. $\mathrm{Hg}$.

Comment.-The history of this patient suggests that he had an aneurysm inside the cavernous sinus and that it extended sufficiently upwards to implicate the right optic nerve. Presumably thrombosis occurred and a spontaneous cure was the result. Ligature of the carotid artery was probably unnecessary though justified by the arteriogram.

Saccular aneurysms of the internal carotid artery inside the anterior part of the cavernous sinus do not appear to interfere with the optic nerve or its blood supply so long as they remain strictly intracavernous. Jefferson (1938) reported seven such cases in each of which vision was unimpaired.

\section{Discussion}

Ophthalmologists have shown an increasing tendency to attribute visual defects to interference with the blood supply rather than to direct pressure on the nerve fibres. It is difficult however to envisage a lesion which would press sufficiently on the blood vessels on the surface of the optic nerve without at the same time pressing on the nerve fibres. On the other hand any effective pressure on the nerve must affect the vessels. The possibility of obstruction to the vessels before they reach the nerve is examined in this paper.

There appears to be general agreement that the tumour which raises or depresses the optic nerve close to the optic foramen is particularly liable to be associated with a central scotoma. It has been pointed out above that a tumour in this situation which either presses the optic nerve downwards or pushes the internal carotid artery upwards might kink or obstruct the ophthalmic artery or its intra- cranial branches. In this way the blood supply of a segment of the macular bundle might be impaired. The blood supply of the intracranial and intracanalicular portions of the bundle is said to be poor when compared with that of the rest of the optic nerve, and this relative poverty has been evoked to explain the alleged vulnerability of the macular fibres.

The hypothesis of hypersensitivity to pressure of the papillo-macular bundle fails to explain why demonstrable pressure on the nerve should in one case produce a purely peripheral defect and in another a defect of a purely central type, nor does it explain why the site and direction of pressure seem to be significant.

On the hypothesis of vascular obstruction the presence or absence of a central scotoma would depend on whether or not a particular vessel or group of vessels were involved. An obstruction to blood vessels has not been demonstrated at operation nor has it been shown by arteriography. As the shadow of the ophthalmic artery fails to appear in many good arteriograms its absence cannot be taken to imply obstruction to that vessel. When the internal carotid artery is occluded proximal to its ophthalmic branch the effect on vision varies with the nature of the occlusion. In spontaneous closure due to thrombosis vision in the corresponding eye is lost, whereas surgical ligation leaves vision unaffected (Walsh and King, 1942). The ophthalmic artery itself has been ligatured in cases of carotid-cavernous aneurysm without producing any visual defects. No opinion can be offered on the effect of partial obstruction to the ophthalmic artery. Lillie and Adson (1934) reported two cases of callus formation in the optic canal associated with a central scotoma. It is probable that whatever the pressure on the nerve itself in these cases, there was some interference with the blood flow through the vessel or through the branches arising in the canal.

Although the presence of a central scotoma directs attention to the optic nerve close to the optic foramen, exploration may not reveal any lesion in this situation. Some years ago McConnell and Mooney (1938) reported a case of subchiasmal aneurysm associated with a definite central scotoma in the right visual field and one for small red objects only in the left. At operation the optic nerves and the chiasma appeared normal in every respect. In this case some factor other than direct pressure must have been concerned in the production of the central scotoma.

Appearances at operation in the majority of cases cannot but suggest that the obvious pressure on the nerve is related to the scotomatous defect, 
but our observations and some of those recorded in the literature suggest that the problem is of a more complex nature.

\section{Summary}

Current theories on the causation of central scotomata associated with space-occupying intracranial lesions are reviewed. The possibility of pressure on the intracranial portion of the ophthalmic artery as a factor in the production of scotomata is discussed.

\section{REFERENCES}

Beck, D. J. K. (1947). Bristol Med-Chir. J., 64, 11. Behr, C. (1935). V. Graefes Arch. Ophthal., 134, 227. Henderson, W. R. (1939). Brit. J. Surg., 26, 811.

Hughes, E. B. C. (1943). Trans. Ophthal. Soc. U.K., $63,143$.

(1945). Ibid., 65, 35.

Jefferson, G. (1937). Brain, 60, 444.

(1938). Brit. J. Sury., 26, 267.

(1940). Proc. R. Soc. Med., 33, 433.

(1945). Doyne Lecture. Trans. Ophth. Soc. U.K., $65,262$.
Kennedy, Foster (1911). Amer. J. med. Sci., 142, 355. (1916). J. Amer. med. Ass., 67, 1361.

Kestenbaum, A. (1947). "Clinical Methods of Neuroophthalmologic examination." London. p. 78.

Larsson, S., and Nord, B. (1947). Nord. Medicin., 34, 1059.

Lillie, W. I., and Adson, A. W. (1934). Arch. Ophthal., Tण Chicago, 12, 500.

Mooney, A. J. (1945). Trans. Ophthal. Soc. U.K., 65, 号 394.

McConnell, A. A., and Mooney, A. J. (1938). Brain, 61, 37.

Paton, Leslie (1909). Ibid., 32, 65.

Peter, L. C. (1931). "Principles and Practice of Perimetry." Philadelphia. p. 230.

Traquair, H. M. (1946). "An Introduction to Clinical Perimetry." London.

Turner, J. W. A. (1973). Brain, 66, 140.

Vail, D. (1948). Amer. J. of Ophthal., 31, 1.

Vincent, C., Hartmann, E., and Le Beau, J. (1938). Z Zbl. §ٌ Neuroschir., 3, 145.

Walsh, F. B., and King, A. B. (1942). Arch. Ophthal., $\vec{\circ}$ Chicago, 27, 1.

Wolff, E. (1939). Trans. Ophth. Soc. U.K., 59, 157.

- (1948). "The Anatomy of the Eye and Orbit." London.

Woods, A. C. (1948). Amer. J. of Ophthal., 31, 1063.

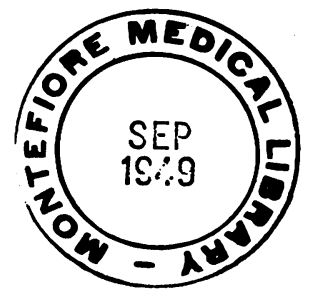

